\title{
Simulation of Impurity Transport and Deposition in the Closed Helical Divertor in the Large Helical Device*)
}

\author{
Mamoru SHOJI $^{1)}$, Gakushi KAWAMURA ${ }^{1,2)}$, Juri ROMAZANOV ${ }^{3)}$, Andreas KIRSCHNER ${ }^{3)}$, \\ Alina EKSAEVA ${ }^{3)}$, Dmitry BORODIN ${ }^{3)}$, Suguru MASUZAKI ${ }^{1,2)}$ and Sebastijan BREZINSEK ${ }^{3)}$ \\ ${ }^{1)}$ National Institute for Fusion Science, National Institutes of Natural Sciences, 322-6 Oroshi-cho, Toki 509-5292, Japan \\ ${ }^{2)}$ The Graduate University for Advanced Studies, SOKENDAI, Shonan Village, Hayama 240-0913, Japan \\ ${ }^{3)}$ Forschungszentrum Jülich GmbH, Institut für Energie- und Klimaforschung - Plasmaphysik, Partner of the Trilateral
} Euregio Cluster (TEC), Jülich 52425, Germany

(Received 16 November 2020 / Accepted 14 December 2020)

\begin{abstract}
Long pulse discharges in the Large Helical Device have often been interrupted by large amounts of dust particle emission from the divertor region caused by the exfoliation of carbon-rich mixed material deposition layers. The plasma wall interaction code ERO2.0 has provided the simulation results of the three-dimensional distribution of the carbon flux density in the divertor region which is quite reasonable with the observed distribution of the carbon-rich deposition layers. The code has also succeeded in reproducing the reduction of the carbon deposition layers on dome plates by changing the target plate configuration in the divertor region. The ERO2.0 simulations have also successfully explained dust particle emission from the inboard side near the equatorial plane for the new target plate configuration at the termination of a long pulse discharge. These simulation results prove that the ERO2.0 code is applicable to predicting the possible position from where the dust particles are released, and to designing an optimized divertor configuration for performing stable long pulse discharges with controlled dust particle emission.
\end{abstract}

(c) 2021 The Japan Society of Plasma Science and Nuclear Fusion Research

Keywords: ero2.0, plasma wall interaction, simulation, impurity transport, divertor, peripheral plasma, emc3eirene, large helical device

DOI: $10.1585 /$ pfr. 16.2403004

\section{Introduction}

The influence of dust particles in magnetic plasma confinement devices has recently gained attention from the viewpoint of the effect on the plasma performances. Long pulse plasma discharges in the Large Helical Device (LHD) provide unique opportunities for studying plasmawall interactions under a steady-state operational condition such as future nuclear fusion reactors [1]. Since the fiscal year (FY) 2010, the divertor configuration in the inboard side of the torus has been replaced to a closed helical divertor from the original open divertor, which is for controlling the peripheral plasma by enhancing neutral particle pumping efficiency due to the higher neutral particle pressure in the closed divertor region [2,3]. Recently, it has been found that long pulse discharges are often interrupted by the large amounts of dust particle emission from the closed helical divertor region [4]. The traces of the exfoliation of carbon-rich mixed material deposition layers were found in the divertor region, which led to the interruption of the long pulse discharges due to radiation collapse caused by the impurities included in the dust particles released from the deposition layers. Two possible

author's e-mail: shohji.mamoru@nifs.ac.jp

*) This article is based on the presentation at the 29th International Toki Conference on Plasma and Fusion Research (ITC29). scenarios of the exfoliation of the deposition layers indicate that the thick carbon-rich layers are easily peeled off from brittle iron-rich layers [5]. For controlling the exfoliation of the deposition layers, the distribution of the carbon flux density in the divertor region was investigated using the Monte-Carlo based three-dimensional plasma wall interaction simulation code ERO2.0 [6]. This analysis can contribute to sustaining stable long pulse discharges with reduced dust particle emission by controlling the carbon flux density in the divertor region.

\section{Interruption of Long Pulse Discharges by Dust Particle Emission from the Divertor Region}

In the experimental campaign in FY2013, a long pulse discharge has been successfully sustained for approximately 48 minutes. The termination process of this plasma discharge was observed with a fast framing camera, which revealed that the plasma was interrupted by radiation collapse induced by emission of the large amounts of dust particles from the closed helical divertor region in the inboard side of the torus [4]. After this experimental campaign, the traces of the exfoliation of deposition layers were found on the surface of dome plates in the divertor region near a lower port, which is shown in Fig. 1 (a). This figure indi- 
(a) Original Configuration

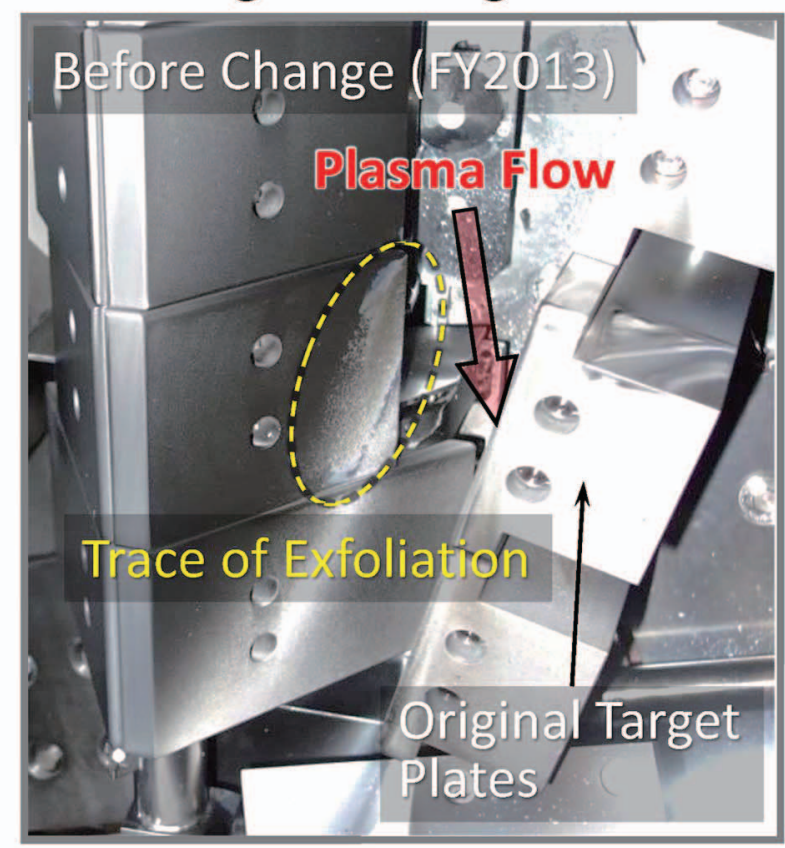

(b) New Configuration

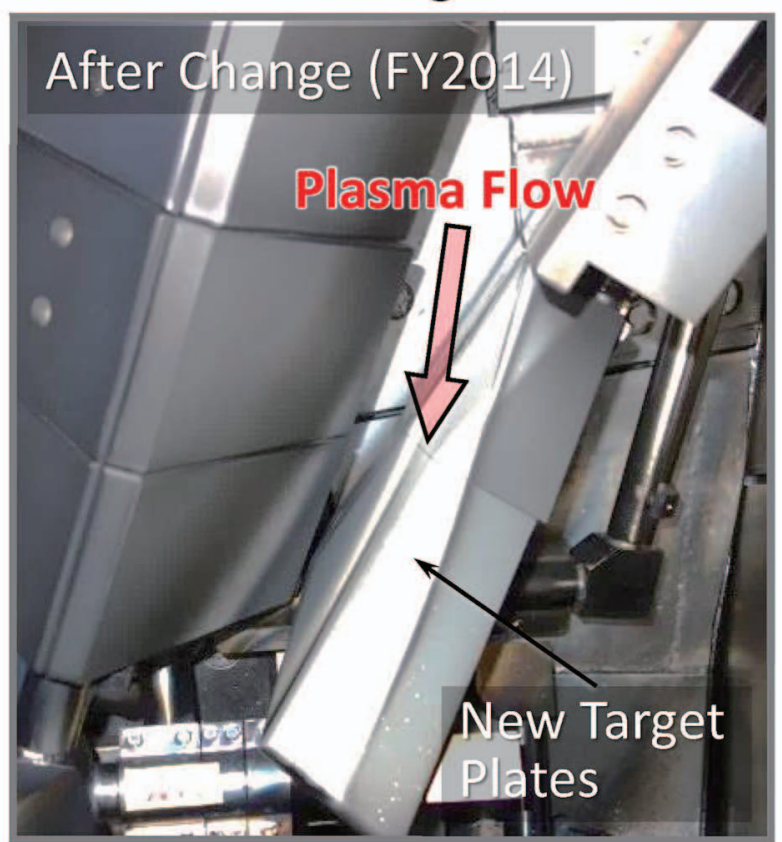

Fig. 1 The image of the carbon-rich mixed material deposition layers on the surface of the dome plates in front of the target plates in the original target plate configuration in FY2013 (a) and that in the new target plate configuration in FY2014 (b). The direction of the plasma flow along the magnetic field lines onto the target plates installed at the lower edge of the closed helical divertor region is schematically indicated as transparent red arrows.

cates that the dust particles were released by the exfoliation of the deposition layers on the dome plates. The closed helical divertor consists of the following three plasma facing components: divertor plates, dome plates, and target plates. The target plates are specially designed divertor plates which are installed close to the dome plates at the upper and lower ends of the closed helical divertor region illustrated in Fig. 2. These plates directly intersect LHD divertor legs and the front surface of these plates directly faces toward the dome plates. Since all the three divertor components are made of isotropic graphite (carbon), the closed divertor locally enhances the carbon deposition on the surface of the dome plates in front of the target plates by physical and chemical sputtering [6].

In order to reduce the carbon deposition on the dome plates, the configuration of the target plates was changed after the experimental campaign in FY2013 (before the experimental campaign in FY2014) such that the front surface of the target plates directly faces toward the main plasma. This change has successfully reduced the deposition of the sputtered carbon on the dome plates, which suppressed the interruption of the long pulse discharges in FY2014 caused by dust particle emission from the dome plates in front of the target plates. Traces of the exfoliation of the deposition layers were not found in the divertor region in this experimental campaign (FY2014), which is shown in Fig. 1 (b).

\section{Setup of the Grid Model of the Closed Helical Divertor for the ERO2.0 Simulation}

The Monte-Carlo based three-dimensional plasmawall interaction simulation code ERO2.0 was applied to investigating the effect of the change of the target plate configuration on the distribution of the carbon flux density on the dome plates in the closed helical divertor region. Figure 2 (a) illustrates the perspective view of the three-dimensional grid model for the ERO2.0 simulation. This model includes the plasma facing components (PFCs) and the vacuum vessel in one helical coil pitch angle $\left(36^{\circ}\right.$ in toroidal direction). The surfaces of the PFCs and the vacuum vessel are treated as carbon and iron, respectively. These components consist of aggregates of many small triangle surfaces which size is less than a few centimetres. The poloidal planes at both toroidal edges are treated as periodic boundaries. Two grid models were constructed for simulating the closed helical divertor configuration before and after the change of the target plates. These are named as original and new target plate configurations, respectively. Figures 2 (b) and (c) display the enlarged figures of the grid model for the original and new target plate configurations, respectively. The target plates in both configurations are shown by areas enclosed by broken black lines. The new target plate configuration (shown in Fig. 2(c)) aims to reduce the plasma heat load on the target plates 


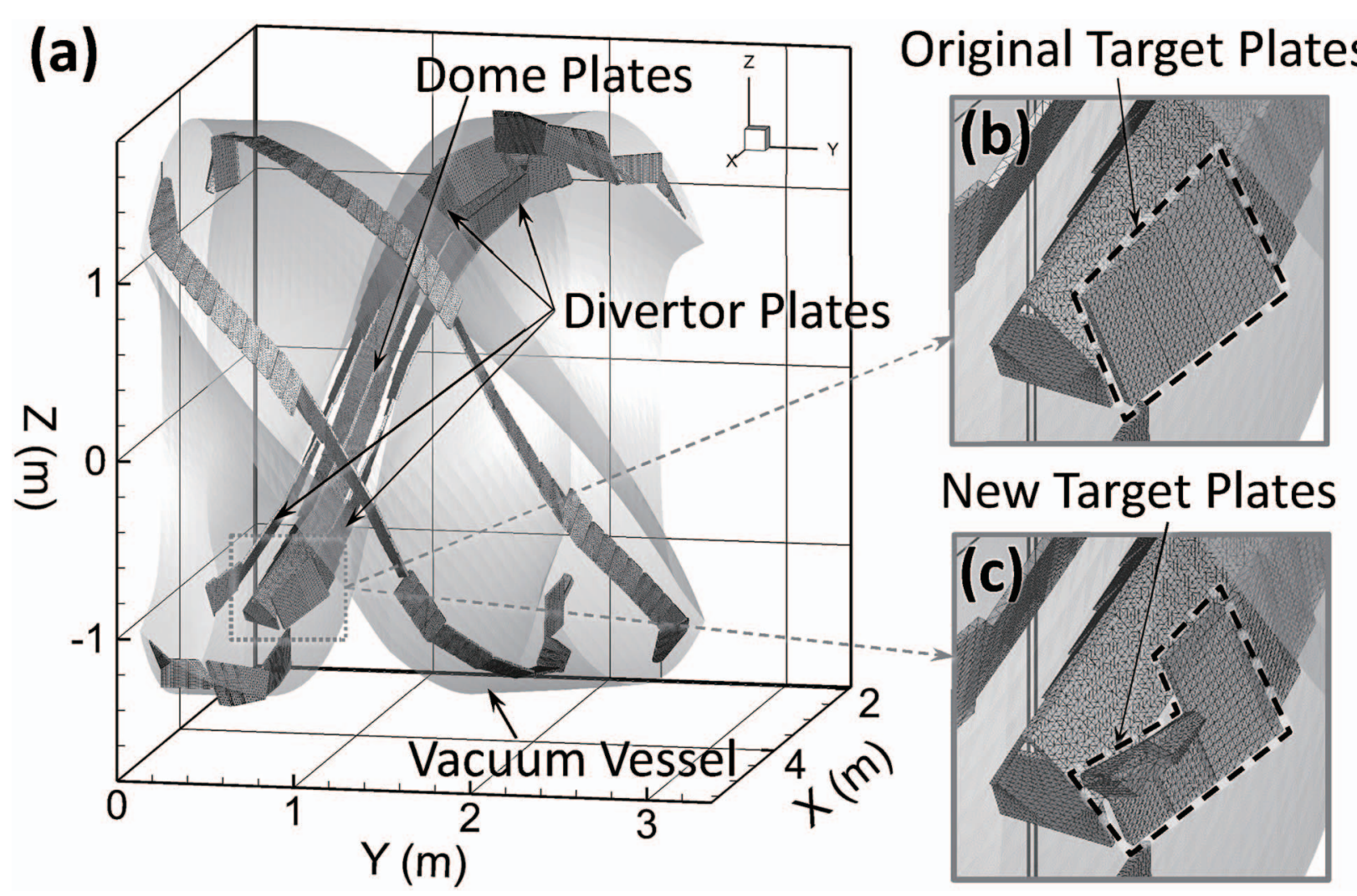

Fig. 2 (a) The perspective view of the three-dimensional grid model for the ERO2.0 simulation. The enlarged figures of the lower divertor components for the original target plate configuration (b) and the new target plate configuration (c). The target plates in both configurations are shown by areas enclosed by black broken lines in Figs. (b) and (c).

by extending the plasma wetted area on the plates, and the control of the carbon deposition on the dome plates in front of the target plates by changing the direction of the sputtered carbon on the target plates. Although the new target plate configuration is advantageous for suppressing the deposition, the pumping efficiency of neutral particles near the target plates decreases due to the lowered neutral particle pressure by the mitigated closure of the divertor configuration.

The fixed three-dimensional background (BG) plasma parameter profile for the ERO2.0 simulation is provided by a three-dimensional edge plasma simulation code (EMC3EIRENE) [7-9], in which a pure deuterium BG plasma is assumed for the simplicity. The plasma parameter profile is the calculation in the case where the plasma heating power $P^{\mathrm{LCFS}}$ and the plasma density $n_{\mathrm{e}}^{\mathrm{LCFS}}$ at an inner boundary of the calculation domain just inside of the LCFS (Last Closed Flux Surface) are set to be $8 \mathrm{MW}$ and $8 \times 10^{19} \mathrm{~m}^{-3}$, respectively. The magnetic configuration is the most typical configuration where the radial position of the magnetic axis $R_{\mathrm{ax}}$ is $3.60 \mathrm{~m}$, and the toroidal magnetic field direction is clockwise (drift effects are not included for calculating the BG plasma parameters in the EMC3-EIRENE code). The perpendicular particle and the ion/electron thermal diffusion coefficients of the BG plasma are assumed to be 0.5 and $1.0 \mathrm{~m}^{2} / \mathrm{s}$, respectively, which are typical values for explaining the measured electron temperature and density profiles in the peripheral plasma in LHD.

In the ERO2.0 code, many test particles (in the order of several ten million), which are representative of carbon atoms or hydrocarbon molecules, are launched from the strike points on the divertor plates. These are produced by physical and chemical sputtering on the divertor plates by incident deuterium ions in the divertor plasma. The ionization/recombination rates of carbon atoms/ions and hydrocarbon molecules/ions in the peripheral plasma are derived from the database on the Atomic Data and Analysis Structure (ADAS) [10]. The ERO2.0 code follows the particles in three-dimensional with the full resolution of the gyromotion of impurity ions without the guiding centre approximation. The code tracks carbon ion trajectories in the plasma with a perpendicular particle diffusion coefficient of $1.0 \mathrm{~m}^{2} / \mathrm{s}$ which provides reasonable simulation results being compatible with both observed carbon line emission ratios and absolute carbon line emission [11]. When the test particles (carbon ions) reach the inner boundary of the grid model, new test particles are regenerated at random positions on the surface at the inner boundary. This is an adequate measure for simulating the impurity transport in the core plasma because of the formation of the nested magnetic flux surfaces in this region. 

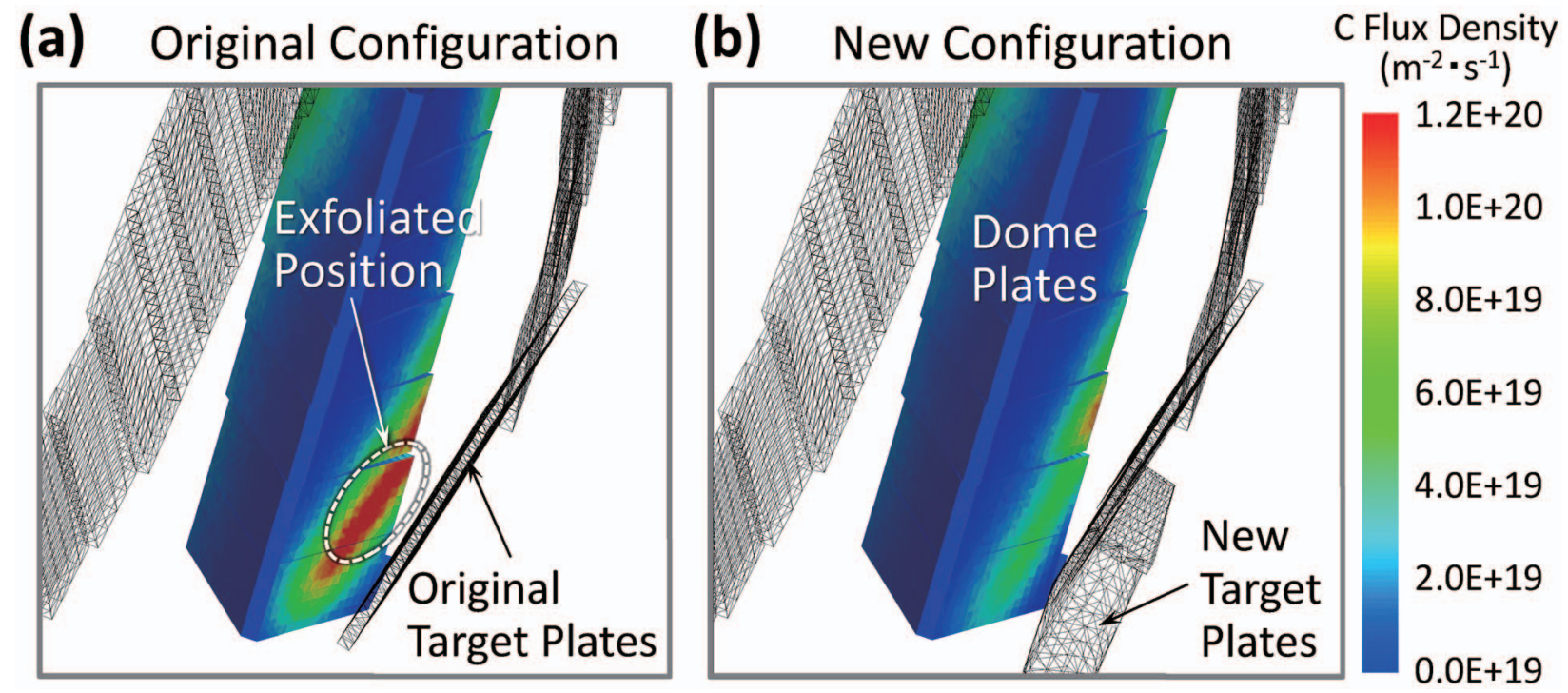

Fig. 3 The simulations of the distribution of the carbon flux density on the dome plates in the closed helical divertor for the original target plate configuration (a) and the new target plate configuration (b).

\section{The ERO2.0 Simulation of the Carbon Flux Density in the Closed Helical Divertor}

In the ERO2.0 simulation, carbon atoms/ions and hydrocarbon molecules/ions colliding with the surface of the PFCs are deposited on or reflected from the surfaces of the PFCs. These particles also induce the physical and chemical sputtering on the PFCs. The angular and energy dependences of the reflection coefficients and sputtering yields are calculated using the database of the calculations by PWI simulation codes such as SDTrimSP [12]. The erosion of the deposited carbon by the BG plasma (deuterium ions) and the sputtered carbon atoms released from the PFCs is not included in this simulation. The re-deposition of the eroded carbon and the carbon self-sputtering are not considered for the simple calculation, which means that this simulation corresponds to a so-called "the first-time step calculation" in the ERO2.0 code.

Figures 3 (a) and (b) give the simulation results of the distribution of the carbon flux density on the surface of the dome plates in the closed helical divertor region for the original and the new target plate configurations, respectively. The ERO2.0 simulations show the reduction of the carbon flux density by changing the target plate configuration, which is consistent with the observed deposition profile of the carbon-rich layers shown in Figs. 1 (a) and (b). This reduced carbon flux density is favourable for controlling the dust emission from the divertor region. The simulation for the original target plate configuration (Fig. 3 (a)) shows that the area with the highest carbon flux density on the dome plates just corresponds to the position where the traces of the exfoliation of the deposition layers were actually found after the experimental campaign in FY2013 (before the change of the target plate configura- tion). This correspondence indicates that the ERO2.0 code exactly predicts a possible position where the deposition layers are exfoliated in the long pulse plasma discharges.

\section{Dust Particle Emission Near the Equatorial Plane in the New Target Plate Configuration}

Long pulse discharges in FY2014 (after the change of the target plate configuration) were often interrupted by dust particle emission from the closed helical divertor region near the equatorial plane in the inboard side of the torus. Figure 4 gives the sequential images showing the interruption process of a long pulse discharge in this experimental campaign (FY2014). These images were taken with a CCD camera installed in an outer port (9-O) for monitoring the closed helical divertor installed in the inboard side of the torus. The observed images reveal that the long pulse discharge was terminated by the emission of the large amounts of dust particles released from the closed helical divertor region. Figure 4 (a) elucidates that the plasma termination was triggered with a bright spot at a position near the equatorial plane in the inboard side.

After the experimental campaign in FY2014, the traces of the exfoliation of the carbon-rich deposition layers were found on the surface at the edge of the dome plates installed near the equatorial plane, which is displayed in Figs. 5 (a) and (b). This exfoliated area exactly agrees with the position where the bright spot was detected with the CCD camera just before the termination of the long pulse discharge (shown in Fig. 4 (a)).

For investigating the reason for the dust particle emission from the divertor region near the equatorial plane in the new target plate configuration, the ERO2.0 simulations 

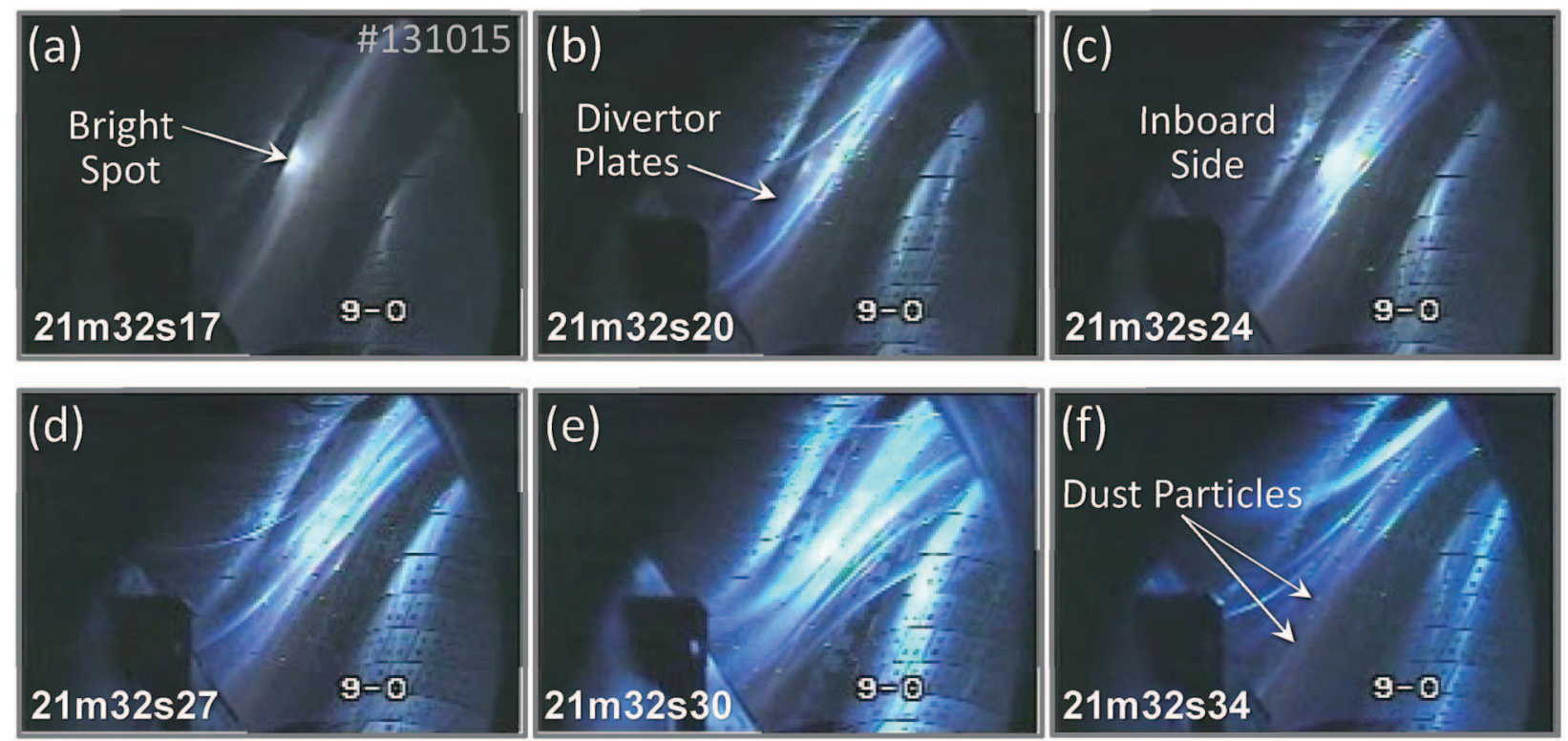

Fig. 4 The sequential images showing the interruption process of a long pulse discharge in the experimental campaign in FY2014.

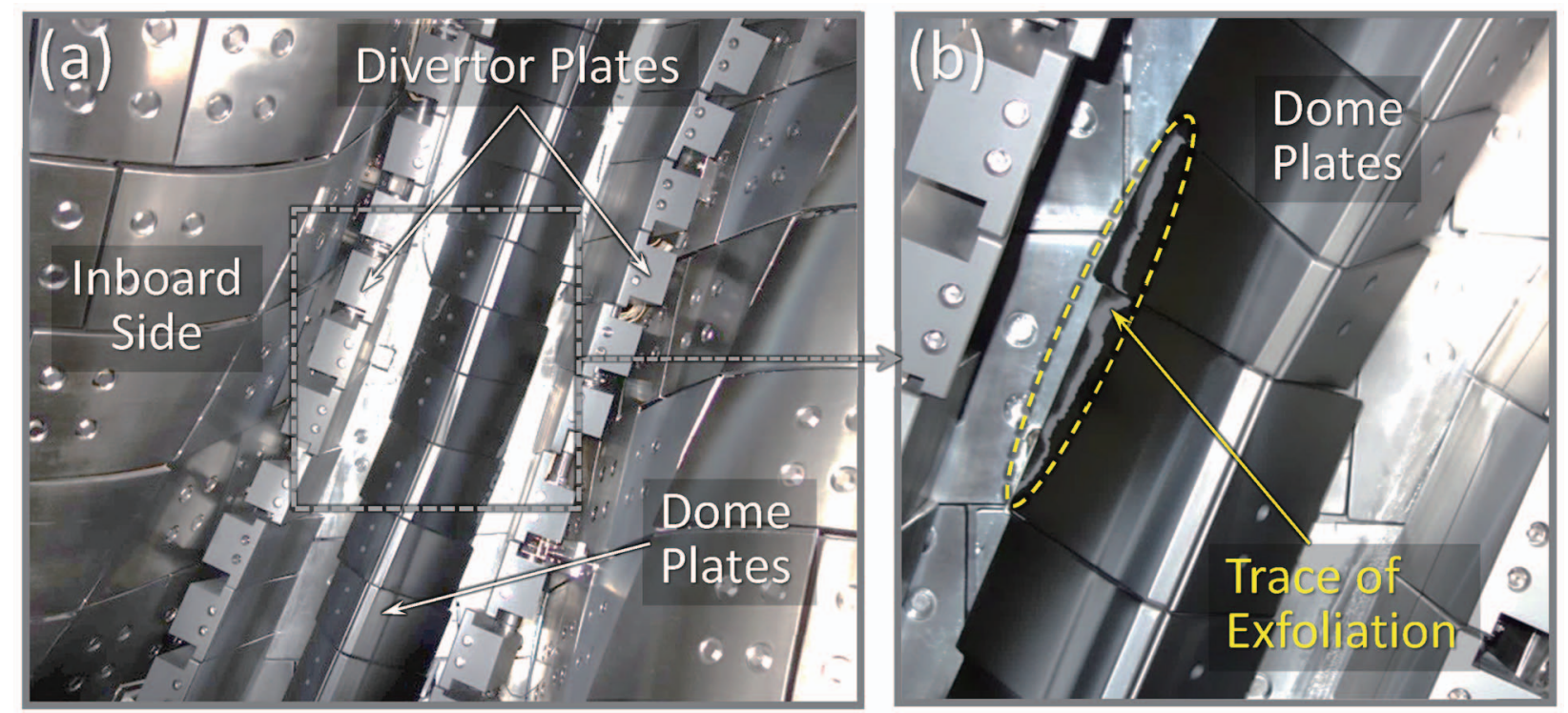

Fig. 5 (a) The images of the trace of the exfoliated carbon-rich deposition layers at the edge of the dome plates near the equatorial plane after the experimental campaign in FY2014 (in the new target plate configuration). (b) A magnified view of the dome plates at the equatorial plane for showing the exfoliation of the deposition layers at the edge of the dome plates.

of the three-dimensional distribution of the carbon flux density on the dome plate are compared in the original and the new target plate configurations. Figures 6 (a) and (b) display the perspective view of the calculated carbon flux density distribution on the dome plates in the original and new target plate configurations, respectively. In the original configuration, the carbon flux density is maximum in front of the target plates, which is shown in the lower part in Fig. 6(a). The carbon flux density near the equatorial plane is the second highest in this configuration. In the new target plate configuration, the carbon flux density is maximum at the edge of the dome plates near the equato- rial plane, in which the carbon flux density in front of the target plates is significantly reduced. These two simulations clearly show that the ERO2.0 simulations reasonably explain the position of the dust particle emission near the equatorial plane in the new target plate configuration observed in the long pulse discharge in FY 2014. This is because thicker carbon-rich deposition layers, which are easily exfoliated from the brittle iron-rich layers, are formed near the equatorial plane than in front of the target plates in the new target plate configuration. 
(a) Original Configuration

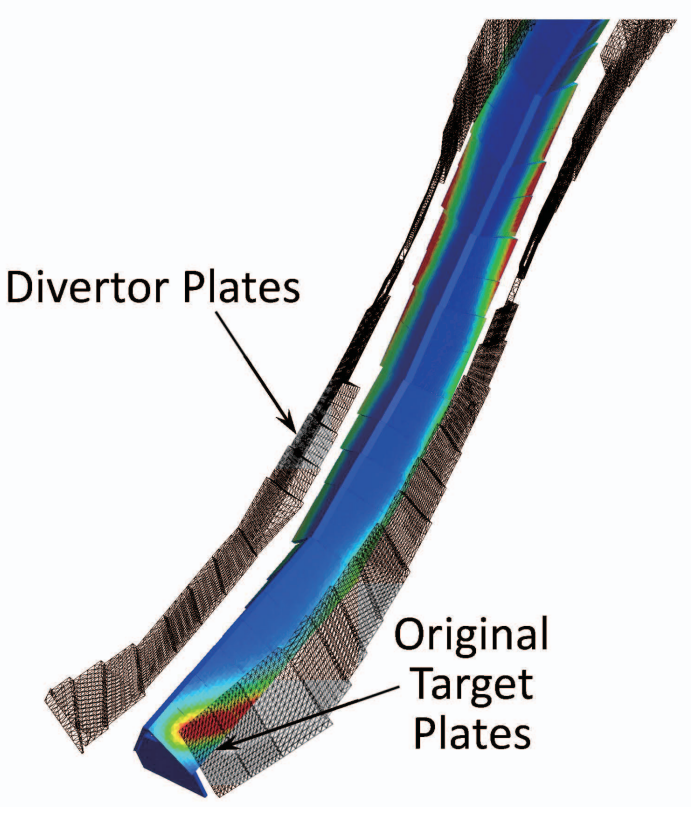

(b) New Configuration

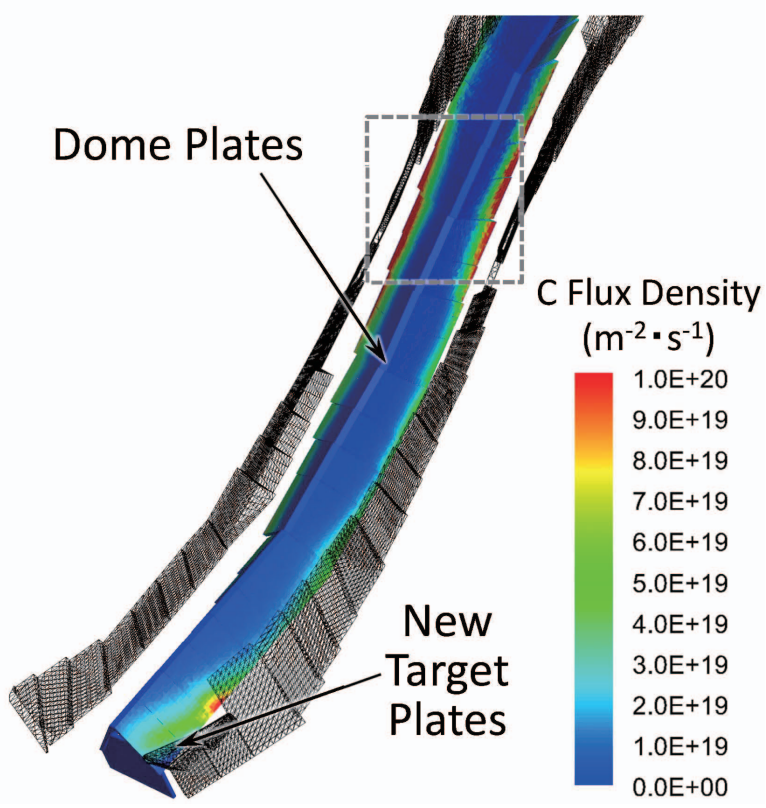

Fig. 6 The ERO2.0 simulations of the carbon flux density distribution on the surface of the dome plates in the original target plate configuration (a) and in the new target plate configuration (b). The grey broken square in Fig. (b) corresponds to the position of the area in Fig. 5 (b).

\section{Summary}

Long pulse plasma discharges in the Large Helical Device have often been interrupted by the large amounts of dust particle emission from the closed helical divertor region by the exfoliation of the carbon-rich mixed material deposition layers. The Monte-Carlo based threedimensional plasma wall interaction code ERO2.0 was applied to the investigation of the carbon flux density distribution in the closed helical divertor. The simulations using ERO2.0 in a background plasma provided by EMC3EIRENE have successfully reproduced the carbon flux density distribution on the dome plates in the closed helical divertor region. In the original target plate configuration (in FY2013), the simulation result of the highest carbon flux density area exactly corresponds to the position where the exfoliation of the deposition layers was found. The simulation also reproduced the significant reduction of the carbon flux density on the surface of the dome plates in front of the target plates in the new configuration (in FY2014). While the change of the target plate configuration has successfully suppressed dust particle emission from the dome plates in front of the target plates installed in the upper and lower ends of the closed divertor region, long pulse discharges were often terminated by dust particle emission from the divertor region near the equatorial plane. This experimental result is also reasonably explained by the ERO2.0 simulation which shows the highest carbon flux density at the edge of the dome plates near the equatorial plane. The experimental results prove that the ERO2.0 code is applicable to predicting the possible po- sition from where the dust particles are released, and to the design of an optimized divertor configuration for sustaining stable long pulse discharges with suppressed dust particle emission.

\section{Acknowledgments}

This work is performed under the auspices of the NIFS Collaboration Research program (NIFS12KNXN236). One of the authors (M.S.) would like to thank Y. Feng for permission to use the EMC3-EIRENE. He also appreciates the computational resources of the LHD numerical analysis server and the plasma simulator in NIFS. This work is also supported by JSPS KAKENHI Grant Numbers $18 \mathrm{H} 01203,16 \mathrm{H} 04619$, and 16K18340.

[1] Y. Takeiri et al., Nucl. Fusion 57, 102023 (2017).

[2] M. Shoji et al., J. Nucl. Mater. 415, S557 (2011).

[3] S. Masuzaki et al., Plasma Fusion Res. 6, 1202007 (2011).

[4] M. Shoji et al., Nucl. Fusion 55, 053014 (2015).

[5] M. Tokitani et al., J. Nucl. Mater. 463, 91 (2015).

[6] J. Romazanov et al., Nucl. Mater. Energy 18, 331 (2019).

[7] Y. Feng et al., Plasma Phys. Control. Fusion 44, 611 (2002).

[8] G. Kawamura et al., Contrib. Plasma Phys. 54, 437 (2014).

[9] G. Kawamura et al., Plasma Phys. Control. Fusion 60, 084005 (2018).

[10] The ADAS User Manual (version 2.6) http://adas.phys. strath.ac.uk/ (2004).

[11] S. Dai et al., Nucl. Fusion 56, 066005 (2016).

[12] W. Möller et al., Comput. Phys. Commun. 51, 355 (1988). 